Alexander Roßnagel

\title{
Quantifizierung der Persönlichkeit - aus grundrechtlicher und datenschutzrechtlicher Sicht
}

\section{Zusammenfassung}

Die Quantifizierung von Persönlichkeitsmerkmalen nimmt durch die Digitalisierung in Form von Profiling, Scoring und statistischen (Big Data-)Mustern zu und verursacht neue gesellschaftliche und rechtliche Herausforderungen. Der Beitrag untersucht, inwiefern diese Quantifizierung den Menschen zu einem Informationsobjekt degradiert und dadurch die Menschenwürde verletzt. Er analysiert und bewertet, inwiefern Profiling und Scoring nach der DatenschutzGrundverordnung zulässig sind, und prüft, inwiefern solche Quantifizierungen erwünschte Differenzierungen oder unerwünschte Diskriminierungen zum Ausdruck bringen. Schließlich prüft er, inwiefern durch solche Quantifizierungen direkt oder indirekt das individuelle und kollektive Verhalten gesteuert werden kann. Der Beitrag schließt mit rechtspolitischen Schlussfolgerungen, die umgesetzt werden müssen, wenn individuelle und gesellschaftliche Freiheit in der digitalen Welt gewahrt werden sollen.

\section{Quantifizierung als Problem des Grundrechts- und Datenschutzes}

Der Beitrag deutet einführend an, welche Herausforderungen für Gesellschaft und Recht durch die zunehmende Quantifizierung der Persönlichkeit entstehen, und untersucht dann vier durch sie hervorgerufene Rechtsfragen des Grundrechts- und Datenschutzes. Diese Untersuchung knüpft zum einen an der Qualifizierung von Menschen durch ihre Quantifizierung und ihrer Degradierung zum Informationsobjekt an und verfolgt die Rechtsfrage, ob die Ersetzung oder die Repräsentation von Menschen durch Zahlen die Menschenwürde verletzt. Die quantitativen Angaben werden - insbesondere im Umgang mit Verbraucherinnen - oft zu Persönlichkeitsprofilen zusammengeführt und zu Score-Werten aggregiert. Daher gilt die zweite Rechtsfrage den Herausforderungen, die damit für die informationelle Selbstbestimmung und den Datenschutz verbunden sind. Vielfach führen Profile und Score-Werte zu quantifizierten Entscheidungen über Menschen und erfordern dann als dritte Rechtsfrage eine Bewertung, ob sie sinnvolle Differenzierungen oder unzulässige Diskriminierungen darstellen. 
Das vierte Rechtsproblem ist dann, wie man aus rechtlicher Sicht damit umgeht, dass die zunehmende Quantifizierung soziale Folgen generiert, die nicht unmittelbar beabsichtigt sein müssen, sondern die einfach als Wirkungen festzustellen sind. Diese quantifizierte Verhaltenssteuerung erzeugt neue Herausforderungen für den Freiheitsschutz. Abschließend hält der Beitrag zu allen vier Rechtsfragen fest, dass sie staatliche Schutzpflichten zum Schutz der Freiheit hervorrufen.

\section{Erscheinungsformen der Quantifizierung}

In entwickelten Gesellschaften gibt es schon immer Formen, Menschen genau $\mathrm{zu}$ erfassen und von anderen $\mathrm{zu}$ unterscheiden. Vielfach erfolgt diese Unterscheidung durch die individuelle Zuordnung von Zahlen. Dies hatte seinen Grund vor allem darin, entweder Menschen zu identifizieren und ihnen bestimmte Entscheidungen zuzuordnen oder sie zu bewerten und in bestimmte Kategorien einzuordnen. An diese Formen der Quantifizierung von Persönlichkeit haben sich alle weitgehend gewöhnt. Die Quantifizierung von Persönlichkeit dringt im Rahmen der Digitalisierung jedoch in alle Gesellschaftsbereiche vor und verfolgt den Zweck, individuelles und kollektives Verhalten vorherzusagen und zu beeinflussen. Hier drängen sich neue Fragen auf, wie diese Entwicklung rechtlich zu bewerten und zu regeln ist.

\subsection{Identifizierung}

Menschen identifizieren sich ständig gegenseitig - man will wissen, mit wem man es zu tun hat, will den anderen wiedererkennen und zuordnen können. Dies erfolgt im näheren sozialen Umfeld mit dem Vornamen oder im weiteren Sozialbereich mit dem Nachnamen. In bürokratischen Zusammenhängen, in denen eine individuelle Bekanntschaft nicht erwartet werden kann, wird der Mensch durch Zahlen repräsentiert, um ihn eindeutig identifizieren und von anderen Menschen ohne Verwechslungsgefahr unterscheiden zu können. In vielen Fällen wird die Identifizierung durch amtliche oder sonstige Ausweise unterstützt. Als generelles Identifikationsmittel gelten der Personalausweis und der Pass. In jedem bürokratischen Zusammenhang wird eine eigene Nummer zur Identifizierung generiert. Behörden verwenden vielfach die Personalausweisnummer, Arbeitgeber die Personalnummer, Unternehmen nutzen eine Kundennummer, Hochschulen eine Matrikelnummer, die Rentenversicherung eine Rentenversicherungsnummer, jede Kreditanstalt eine Kontonummer und jedes Krankenhaus eine Patientennummer. Auf diese Weise wird jede Person durch 
eine Fülle von Identifikationsnummern repräsentiert, die in allen Fällen die Grundlage sind, sie zu identifizieren, ihr Rechte einzuräumen oder Handlungen zu ermöglichen, und die jeweils der Schlüssel für eine mehr oder weniger umfangreiche Sammlung von Daten über sie sind.

Bereits diese aus der analogen Welt schon lange bekannten Quantifizierungen von Persönlichkeit, die durch sie geformte Bildung von Identität, die mit ihr verbundene mehr oder weniger eng verknüpfte Identifizierung in unterschiedlichen sozialen Handlungsfeldern und die dadurch ermöglichte Überwachung von Handlungsvollzügen verursacht eine Fülle von Fragen des Grundrechts- und Datenschutzes (Hornung/Engemann 2016). Sie wird inzwischen aber kaum noch problematisiert, weil viele sich an die ständige Identifizierung gewöhnt haben, ihre bürokratische Notwendigkeit vielfach einsehen und die mit ihr verbundenen Probleme der Grundrechtsausübung ausblenden.

\subsection{Qualifizierung}

Viel gravierender als die Identifizierung durch Zahlen ist es jedoch, wenn Eigenschaften von Menschen durch Zahlen repräsentiert werden. In diesem Fall wird Qualität durch Quantität ausgedrückt. Dies ist in der Regel der Fall, wenn in Massenverfahren selektiert werden muss und der Entscheider sich auf Bewertungen menschlicher Eigenschaften durch bestimmte andere Stellen stützen will oder verlassen muss. Auch für diese Form der Bewertung menschlicher Eigenschaften und der Weitergabe dieser Bewertungen haben viele Institutionen unterschiedliche Formen von Quantifizierungen entwickelt und nutzen diese breit in unterschiedlichen sozialen Zusammenhängen:

Die Hochschulen entscheiden aufgrund des Abiturzeugnisses und der Schulnoten darüber, ob jemand überhaupt oder ein bestimmtes Fach studieren darf. Das Kreditinstitut oder der Online-Shop entscheiden aufgrund eines Credit-Scores darüber, ob jemand überhaupt einen Kredit oder einen Kredit nur zu bestimmten Bedingungen erhält. Berufungskommissionen entscheiden aufgrund der Publikations- und Zitationsindikatoren, wer zu Berufungsvorträgen eingeladen wird. Diese Quantifizierung von Eigenschaften wird von den betroffenen Personen übernommen: Wohnungsinteressenten bringen zur Wohnungsbesichtigung schon einen Ausdruck ihres Kredit-Scores mit, Professoren publizieren ihre Publikations- und Zitationsindikatoren und ihren Rankingplatz und wirken daran mit, eine Forscherpersönlichkeit auf eine Zahl zu reduzieren.

Mit der Digitalisierung aller Lebensbereiche nimmt aber auch die Quantifizierung aller Persönlichkeitsmerkmale immer mehr zu und wird Grundlage vieler informeller sozialer Zuordnungs- und Auswahlprozesse: Viele Betroffene präsentieren als Maß ihrer Beliebtheit die Zahl ihrer „Likes“, als Maß ihrer Gesundheit körperliche Messwerte und als Maß ihrer Fitness ihren Body-Mass-In- 
dex. Immer mehr Stellen sammeln Daten über andere Personen in zunehmendem Umfang und steigender Dichte und versuchen zugleich, ihre Erkenntnisse über die spezifischen Eigenschaften dieser Personen in jeweils einer Zahl zu aggregieren: Aus Verbraucherprofilen entstehen Konsumenten-Scores, aus Fahrerprofilen Fahrer-Scores und aus Bewerberprofilen Bewerberinnen-Scores (Dzida/Groh 2018). Den Gipfel dieser Entwicklung bilden der chinesische Staat und die chinesischen Internet-Konzerne in Form ihres Social Citizen Scores, in dem sie die gesamte Persönlichkeit in einer einzigen Zahl zusammenfassen. ${ }^{3}$

\subsection{Vorhersage und Steuerung von Verhalten}

Bei der Quantifizierung von Persönlichkeitsmerkmalen geht es immer darum, individuelles und kollektives Verhalten vorherzusagen und das Wissen über Menschen dafür zu nutzen, ihr Verhalten zu steuern. Um diese Macht über andere Menschen ausüben zu können, sammeln Staaten und Unternehmen möglichst viele Daten, werten diese aus, integrieren die Ergebnisse in Persönlichkeitsprofilen und fassen sie zu Zahlenwerten zusammen. Diese bilden dann die Grundlage für bestimmte - möglichst automatisierte - Entscheidungen und beeinflussen nach ihrem Bekanntwerden durch ihre Orientierungsrelevanz indirekt das Verhalten der Menschen im Sinn der verantwortlichen Stellen. Je weiter diese Formen des (verdeckten) Machtaufbaus und der Machtausübung in alle Gesellschaftsbereiche eindringen, desto dringender stellt sich die Frage, inwieweit sie die Verwirklichungsbedingungen von Freiheit und Gleichheit verändern können. Der zeitlichen und funktionalen Abfolge dieses grundrechtsrelevanten Machtaufbaus und der Machtausübung folgt die Gliederung der folgenden Überlegungen.

\section{Qualifizierung durch Quantität}

Wer die Qualität von Persönlichkeitsmerkmalen oder gar der Persönlichkeit eines Menschen auf eine einzige Zahl beschränkt, reduziert eine reichhaltige Persönlichkeit in maximaler Weise. Durch die Quantifizierung geht nahezu alles verloren, was diesen Menschen ausmacht - seine Entwicklung, seine Erfahrungen, seine Befähigungen, seine Interessen, Überzeugungen, Hoffnungen und Erkenntnisse, seine Anstrengungen, Erfolge und Lernprozesse. Für ihn oder sie steht nur noch eine Zahl. Die Aufmerksamkeit wird nur auf sie gelenkt, sie ent- 
scheidet über seine Behandlung, die Erfüllung seiner Anliegen, sogar - wie im Fall der Abiturnote - über seine Berufschancen.

\subsection{Würde des Menschen}

Aus grundrechtlicher Sicht stellt sich die Frage, ob diese extreme Reduktion von Persönlichkeit auf eine einzige Zahl die Menschenwürde beeinträchtigt. Die von Art. 1 GRCh und Art. 1 Abs. 1 GG geschützte Menschenwürde soll die Subjektqualität des Menschen wahren und schützen und fordert deshalb, dessen Individualität, Identität sowie physische, psychische und moralische Integrität zu respektieren. Die Quantifizierung könnte diese Subjektqualität des Menschen auf zwei Ebenen verletzten: zum einen durch Missachtung des Menschen durch die Reduktion auf ein bloßes Informationsobjekt und zum anderen durch seine lückenlose Registrierung und Katalogisierung.

Einen positiven Begriff der Menschenwürde hat die höchstrichterliche Rechtsprechung in der Europäischen Union und in Deutschland nicht entwickelt. Sie füllt ihn vielmehr von der Verletzung her mit Inhalt. Nach der in Deutschland dominierenden „Objektformel ${ }^{\star 4}$ ist die Menschenwürde betroffen, „wenn der konkrete Mensch zum Objekt, zu einem bloßen Mittel, zur vertretbaren Größe herabgewürdigt wird“" (Herdegen 2018). Diese negative Definition richtet die verfassungsrechtliche Prüfung auf die mögliche Erniedrigung des Einzelnen. Danach setzt die Verletzung der Würde des Menschen voraus, dass er einer Behandlung ausgesetzt wird, „die seine Subjektqualität prinzipiell in Frage stellt, oder dass in der Behandlung im konkreten Fall eine willkürliche Missachtung der Würde des Menschen liegt". Diese Missachtung muss „Ausdruck der Verachtung des Wertes“ sein, „der dem Menschen kraft seines Personseins zukommt". 5 Wird der Mensch durch Scoring zum reinen Informationsobjekt, weil staatliche Stellen sich die Arbeit erleichtern und private Unternehmen darüber ihren Gewinn erhöhen, könnte dies als Missachtung seiner Subjektqualität und damit als Verletzung seiner Menschwürde angesehen werden.

Oft liegt der Score-Bildung eine umfassende räumliche und zeitliche Erfassung des Verhaltens sehr vieler Menschen zugrunde. Hierzu hat das Bundesverfassungsgericht für die staatliche Datenerhebung festgestellt: „Mit der Menschenwürde wäre es nicht zu vereinbaren, wenn der Staat das Recht für sich in Anspruch nehmen könnte, den Menschen zwangsweise in seiner ganzen Persönlichkeit zu registrieren und zu katalogisieren, sei es auch in der Anonymität

4 S. z.B. BVerfGE 27, 1 (6); 30, 1 (26); 45, 187 (228); 50, 166 (175); 87, 209 (228); 102, 347 (367); 109, 279 (312f.); 115, 118 (153); 116, 69 (85f.); 117, 71 (89); 131, 258 (286).

5 BVerfGE 87, 228; Jarass (2018), Art. 1 GG Rn. 11. So ist auch das Verständnis der Menschenwürde nach Art. 1 GRCh qu/verstehen s. zB. Jaiass (2016), Art. B GRCh Rn. 8. 
einer statistischen Erhebung, und ihn damit wie eine Sache zu behandeln, die einer Bestandsaufnahme in jeder Beziehung zugänglich ist." ${ }^{\text {"6 }}$ Der Mensch darf nicht „zum bloßen Informationsobjekt" werden. ${ }^{7}$ Aus Grundrechtssicht der betroffenen Person kann es keinen Unterschied machen, ob sie ,bei systematischer Datensammlung zu einem bloßen Objekt staatlicher Stellen oder wirtschaftlicher Marketingstrategen“ wird (Di Fabio 2001). Ein Beispiel für eine Behandlung als bloßes Objekt ist eine zeitlich und räumlich lückenlose Überwachung, weil sie den Menschen dauerhaft in seinem Verhalten manipuliert.

In erster Näherung könnte man zu dem Ergebnis kommen, dass zumindest eine extreme Quantifizierung des Menschen diesen zu einem bloßen Informationsobjekt und zum Gegenstand einer lückenlosen Registrierung und Katalogisierung herabwürdigt und daher eine Verletzung der Menschenwürde darstellt.

\subsection{Verletzung der Menschenwürde?}

Für eine Verletzung der Menschenwürde muss die Reduktion eines Menschen auf ein bloßes Objekt jedoch eine Missachtung der Persönlichkeit zum Ausdruck bringen. Typische Fälle, die die Rechtsprechung als eine solche Verletzung der Menschenwürde anerkannt hat, sind Folter, Sklaverei, Vernichtung ethnischer, nationaler, rassischer oder religiöser Gruppen, Verschleppung, unmenschliche oder erniedrigende Strafen und Behandlungsweisen, vollständige Entrechtung, Vernichtung sogenannten lebensunwerten Lebens und Menschenversuche. ${ }^{8}$ Auch in der Erniedrigung oder Ächtung von Personen ${ }^{9}$ und in der „Kommerzialisierung menschlichen Daseins“ kann eine Beeinträchtigung liegen. ${ }^{10}$

Trotzdem sollte die Feststellung, die Menschenwürde sei verletzt, nicht vorschnell getroffen werden. Die Menschwürde darf - im Vergleich mit ihren anerkannten Verletzungen - nicht ,,in kleiner Münze“ eingefordert werden. Der Höchstwert des Grundgesetzes und der Grundrechtecharta verliert seine fundamentale Bedeutung, wenn er immer wieder benutzt wird, um negative Entwicklungen abzuwehren, die auch unter den Schutz anderer Grundrechte fallen und die mit einer freiheitsförderlichen Politikgestaltung vermieden werden können.

Im Ergebnis sollte also nur in Extremfällen, in denen es um eine langfristige und umfassende Überwachung der wichtigsten sozialen Handlungen oder

6 BVerfGE 27, 1 (6).

7 BVerfGE 65, 1 (48).

8 Hessischer Staatsgerichtshof, Deutsches Verwaltungsblatt 1974, $940 \mathrm{ff}$.; s. hierzu auch die Übersicht bei Kunig (2012), Art. 1 Rn. 36.

9 BVerfGE 102, 347 (367).

10 BVerfGE 96, 375 (400), s/auch Jarass (2018), Art. 1 GG Rn. 13. 
um die Score-Bildung des nahezu gesamten sozialen Verhaltens und der erfassbaren individuellen Fähigkeiten und Einstellungen geht, von einer Verletzung der Menschenwürde gesprochen werden, nicht aber bei jeder bereichsspezifischen Bildung von Score-Werten, die für eventuell partikulare, aber nachvollziehbare Interessen eingesetzt werden.

\subsection{Freie Entfaltung}

Außerdem ist bei der Quantifizierung menschlicher Eigenschaften zu berücksichtigen, dass sie oft von den betroffenen Personen selbst vorgenommen und als Ausdruck einer freien Entfaltung ihrer Persönlichkeit angesehen wird. Dies gilt nicht nur für Hochschullehrende, die stolz ihren Publikationsindex, die Zitierfrequenz eines Aufsatzes oder den Platz in irgendeinem Ranking publizieren. Dies gilt auch für die Anhänger der Quantified Self-Bewegung, die jeweils aktuelle Werte ihrer Körperfunktionen im Internet bekannt geben oder andere Formen des Live Logging und Self Tracking verfolgen. Schließlich kann die Veröffentlichung eines solchen Ergebnisses der eigenen sozialen Reputation dienen und ist letztlich auch ein Ausdruck informationeller Selbstbestimmung. Nicht jeder muss eine solche Form von Selbstentblößung mögen. Aber wer sich selbst gern mit einem Score definiert und darauf Wert legt, dem verbietet das Recht diese Handlungsform nicht - auch nicht sein Grundrecht auf Schutz seiner Menschwürde.

\subsection{Wandel des Begriffs der Menschenwürde?}

Daher stellt sich die Frage, ob bezogen auf die zunehmende Digitalisierung aller Gesellschaftsbereiche nicht auch die Ausdrucksformen der Menschwürde einem gewissen Wandlungsprozess unterliegen. Bezogen auf die Quantifizierung menschlicher Merkmale und Eigenschaften ist entsprechend der Veränderung menschlicher Verhaltensweisen stärker nach ihrem Zweck zu differenzieren. Viele Zwecke der Quantifizierung sind mit der Menschenwürde vereinbar, weil sie für das menschliche Zusammenleben hilfreich sind, weil sie die Handhabbarkeit von Vorgängen im Umgang mit Menschen erleichtern, eine gewünschte soziale Selektion ermöglichen oder zum Ausdruck bringen - ohne verächtlich oder missbräuchlich zu sein. Vor allem ist immer zu prüfen, wo die Argumentation mit der Menschwürde Menschen schützt und wo sie sozial tolerierbares menschliches Verhalten einschränkt. 


\section{Profiling und Scoring}

Das zweite Rechtsproblem der Quantifizierung besteht darin, dass jeder ScoreWertbildung ein Persönlichkeitsprofil zugrunde liegt. Unter Profiling versteht Art. 4 Nr. 4 DSGVO, ,jede Art der automatisierten Verarbeitung personenbezogener Daten, die darin besteht, dass diese personenbezogenen Daten verwendet werden, um bestimmte persönliche Aspekte, die sich auf eine natürliche Person beziehen, zu bewerten, insbesondere um Aspekte bezüglich Arbeitsleistung, wirtschaftliche Lage, Gesundheit, persönliche Vorlieben, Interessen, Zuverlässigkeit, Verhalten, Aufenthaltsort oder Ortswechsel dieser natürlichen Person zu analysieren oder vorherzusagen“. Diese Profile sind in der Regel umso aussagekräftiger, je mehr personenbezogene Daten ihnen zugrunde liegen. Umso mehr relevante Persönlichkeitsmerkmale erfasst und bewertet werden, umso aussagekräftiger und verlässlicher ist der Score-Wert, der aus dem Profil gebildet wird. So können personenbezogene Daten über die Konsuminteressen und -gewohnheiten einer Verbraucherin, ihren Zeitvertreib und ihre Hobbies sowie ihre Vorlieben, Schwächen und Abneigungen erkennen lassen, wie kreditwürdig, zahlungsfähig und zahlungsbereit sie ist und für welche (Werbe-)Argumente, Suggestionen und Versprechungen sie aufnahmebereit ist. Dies kann in einem oder mehreren Scores zusammengefasst die jeweilige (Online-)Kundenansprache steuern (Taeger 2016; Moos/Rothkegel 2016; SVRV 2018). Diese Zusammenhänge verleiten dazu, möglichst viele Daten über Menschen zu erfassen, zu sammeln und auszuwerten.

\subsection{Beschränkung der Selbstbestimmung}

Die Daten gewinnen die Unternehmen durch die unmittelbaren Kontakte zu ihren Kunden, vor allem aber durch ihre Identifizierung und das Tracking ihres Verhaltens - online und offline - außerhalb des eigentlichen Kundenkontakts (Ammicht Quinn u.a. 2018). Sie führen die erfassten Daten zusammen, reichern sie durch statistische Daten und ihre Zusammenhänge an, werten sie durch Big Data-Mechanismen und künftig auch selbstlernende Systeme aus und ordnen sie einem Profil zu. Diese Verarbeitungen personenbezogener Daten sind für die betroffene Person intransparent. Durch dieses Vorgehen entstehen Informationen über sie, die sie nicht preisgeben wollte, die sie vielleicht sogar selbst gar nicht kennt.

Diese intrasparente und nicht beeinflussbare Verarbeitung personenbezogener Daten ist ein besonders tiefer Eingriff in die informationelle Selbstbestimmung der betroffenen Person (SVRV 2018). Durch sie werden Eigenschaften entdeckt und bis dahin unbekannte Informationen über sie für Dritte verfügbar, über deren Preisgabe die betroffene Person nichtentscheidenkonnte. Diese Er- 
kenntnisse werden auf die Person angewendet, ohne dass sie dies weiß und verhindern kann. Ihr ist es unmöglich, die Kontrolle über die über sie im Umlauf befindlichen Daten zu behalten. Informationen, die durch Korrelation und Kombination anderer, vorhandener Daten gewonnen werden, bergen außerdem die Gefahr, unzutreffend zu sein, und kompromittieren die informationelle Selbstbestimmung damit zusätzlich (Roßnagel/Nebel 2015).

\subsection{Beschränkung der Selbstentfaltung}

Diese Form der Datenverarbeitung ist auch ein Eingriff in die Freiheit der Selbstentfaltung der betroffenen Person. Denn es werden immer Daten aus der Vergangenheit benutzt und aus diesen Schlussfolgerungen auf Eigenschaften der betroffenen Person gezogen. Das Profil oder der Score halten diese Eigenschaften fest und legen diese der Behandlung der betroffenen Person zugrunde. Eine selbstbestimmte adressatenspezifische Darstellung in unterschiedlichen sozialen Beziehungen ist nicht mehr möglich.

Die informationelle Selbstbestimmung will aber gerade ermöglichen, dass jeder in unterschiedlichen sozialen Kontexten sich frei darstellen und mit anderen in der jeweiligen Beziehung nach seiner Selbstbestimmung kommunizieren kann. Die damit verbundene Freiheit der Selbstentfaltung soll ihm auch ermöglichen, in einer spezifischen sozialen Beziehung sich neu zu präsentieren, Veränderungen darzustellen und einen ,neuen Anlauf“ zu versuchen. Eine neue Selbstentfaltung wird jedoch unmöglich, wenn man immer wieder auf das Profil und die Scores aus der Vergangenheit reduziert wird.

\subsection{Datenschutzrechtliche Zulässigkeit}

Können Profiling und Scoring datenschutzrechtlich zulässig sein, wenn sie zu einer Einschränkung der Selbstbestimmung und Selbstentfaltung führen? $\mathrm{Zu}$ welchen Zwecken und in welchem Umfang die Verarbeitung personenbezogener Daten zulässig ist, regelt die Datenschutzgrundverordnung (DSGVO) in ihrem Art.6. Für die zulässige Datenverarbeitung durch Verantwortliche aus der Wirtschaft nennt Art. 6 Abs. 1 UAbs. 1 lit. a, b und f DSGVO drei mögliche Gründe:

Der erste Grund ist nach lit. b, dass die Datenverarbeitung für die Erfüllung eines Vertrags mit der betroffenen Person erforderlich ist. Ein Profil zu erstellen und einen Score zu bilden, ist für die Erfüllung eines Vertrags sehr selten notwendig. Eine Ausnahme könnte z.B. ein Vertrag über eine personalisierte Dienstleistung sein. Wer ein virtuelles Assistenzsystem abonniert, das nach den Vertragsbedingungen permanent vieles über den Abonnenten lernen muss, um ihn immer besser beraten und unterstützen zu können, muss damit einverstan- 
den sein, dass dieses Assistenzsystem ein Profil anlegt und durch personenbezogene Daten anreichert. Dies gilt für die Daten, die aus der Abwicklung dieses Vertrags entstehen. Dies gilt aber auch für andere Daten, über die der Verantwortliche rechtmäßig verfügt, weil sie z.B. aus anderen Vertragsverhältnissen stammen (z.B. Mobilfunk oder Internetnutzung). ${ }^{11} \mathrm{Zu}$ prüfen ist jedoch immer, ob die konkrete Verarbeitung der konkreten Daten jeweils tatsächlich notwendig ist, um den Vertrag über eine personalisierte Dienstleistung zu erfüllen.

Der zweite Grund könnte nach lit. f sein, dass die Datenverarbeitung zur Wahrung der berechtigten Interessen des Verantwortlichen oder eines Dritten erforderlich ist und die Interessen der betroffenen Person am Unterlassen der Datenverarbeitung nicht überwiegen. Als „berechtigtes Interesse“ gilt jeder rechtlich zulässige Zweck. Es ist bei ökonomischen Zwecksetzungen im Regelfall anzunehmen. Je nach Bestimmung dieses Interesses kann eine Profilbildung und Score-Bestimmung erforderlich sein. Allerdings dürfen die schutzwürdigen Interessen der betroffenen Person nicht überwiegen. Sie richten sich zum einen nach dem berechtigten Erwartungshorizont der betroffenen Person: Mit welcher Datenverarbeitung muss sie bei einem fairen Verantwortlichen rechnen? Mit der Bildung von Profilen, die aus fremden Daten aus fremden Quellen gewonnen werden oder die aus einem umfangreichen Tracking ihrer Internetaktivitäten stammen, muss sie nicht rechnen. Grundsätzlich muss sie nur mit Datenverarbeitungen rechnen, über die der Verantwortliche sie informiert hat. Verdeckte Datenverarbeitungen führen im Regelfall dazu, dass die schutzwürdigen Interessen am Unterlassen dieser Datenverarbeitung überwiegen. Zweitens richten sich die schutzwürdigen Interessen der betroffenen Person nach dem Maß der Erfassung von Daten und ihrer Auswertung und damit der Tiefe des Eingriffs in ihr Grundrecht auf Datenschutz (Art. 8 Abs. 1 GRCh) und informationelle Selbstbestimmung (Art. 2 Abs. 1 in Verbindung mit Art. 1 Abs. 1 GG). Je detaillierter und je umfassender das Profil ist oder werden soll, umso höher wiegt ihr Interesse, dies zu verhindern. Drittens ist zu berücksichtigen, welche Verwendung des Profils oder des Scores der Verantwortliche intendiert oder durchführt und mit welchen nicht intendierten Folgen die betroffene Person rechnen muss. Schließlich ist relevant, wie aussagekräftig und zutreffend das Profil oder der Score ist. Dies ist letztlich eine Frage der Datenqualität und der Sorgfalt der Datenverarbeitung (s. z.B. §31 BDSG). Betrachtet man diese Bedingungen für die Schutzwürdigkeit der Betroffeneninteressen zusammen, dürften die meisten Verbraucherprofile und Scores nicht mit überwiegenden berechtigten Interessen zu rechtfertigen sein. Ausnahmen könnten sehr zweckbegrenzte und in der Verwendung gesicherte Scores sein, mit deren Be-

11 Diese für Profiling zu verwenden, dürfte aber nach Art. 6 Abs. 4 DSGVO selten mit dem bisherigen Zweck vereinbar sein. 
stehen und Verwendung die betroffene Person rechnen musste und deren Erstellung und Verwendung mit höchster Sorgfalt erfolgt. Individualisierte Werbung oder individualisierte Preisgestaltung auf der Grundlage eines Profils, das Interessen, Präferenzen, Konsum-, Mobilitäts-, Kommunikations- und Bezahlverhalten, Aktivitäten in Social Networks, Kontakte, Wohnort und ähnliche Merkmale umfasst, ist danach nicht zu rechtfertigen.

Dritter Rechtfertigungsgrund wäre nach lit. a eine Einwilligung der betroffenen Person. Diese muss, um wirksam zu sein, informiert, freiwillig und bestimmt erteilt sein und fair genutzt werden. Informiert heißt, dass der Verantwortliche die betroffene Person zuvor über Zweck, Umfang und Detaillierung genau informieren und auch die Bildung eines Scores benennen muss. Dies umfasst nach Art. 13 Abs. 2 lit. f und 14 Abs. 2 lit. g DSGVO auch eine Erläuterung, wie die Score-Bildung erfolgt. Die Einwilligung muss freiwillig sein. Art. 7 Abs. 4 DSGVO verbietet eine Vertragsleistung von einer Einwilligung in eine Datenverarbeitung abhängig zu machen, die für diese Vertragsleistung nicht erforderlich ist. Drittens muss der Text der Einwilligung ausreichend bestimmt sein, sie muss also im Text die Score-Wertbildung erwähnen. Schließlich muss die Einholung der Einwilligung fair erfolgen. Fair ist das Einholen der Einwilligung, wenn die Datenverarbeitung nicht auch auf einen anderen Erlaubnistatbestand gestützt wird. Wenn die Verantwortlichen ihre Datenverarbeitung bereits auf die Erlaubnistatbestände der Vertragsdatenverarbeitung nach lit. b oder der überwiegenden berechtigten Interessen nach lit. f stützen können, verstößt es gegen „Treu und Glauben“ gemäß Art. 5 Abs. 1 lit. a DSGVO, wenn die betroffene Person bei einer Einwilligung keine freie Wahlmöglichkeit über die Datenverarbeitung hat und bei einem Widerruf die weitere Datenverarbeitung nicht verhindern kann. Fordert der Verantwortliche eine Einwilligung, obwohl die Datenverarbeitung durch einen gesetzlichen Erlaubnistatbestand erlaubt ist, missbraucht er das Vertrauen der betroffenen Person. In diesem Fall führt der nach Art. 7 Abs. 3 Satz 3 DSGVO geforderte Hinweis auf ein Widerspruchsrecht in die Irre, wenn bei dessen Ausübung die Datenverarbeitung dennoch fortgeführt wird, weil sie durch den gesetzlichen Erlaubnistatbestand erlaubt ist (Erwägungsgrund 43 DSGVO; Roßnagel 2019; Brink/Hertfelder 2019).

Im Ergebnis dürften Profiling und Scoring - von Kredit-Scoring abgesehen (s. § 31 BDSG) - im Kontext von Wirtschaftsunternehmen datenschutzrechtlich meist nicht zu rechtfertigen sein. 


\section{Quantifizierte Entscheidungen}

Welche soziale und rechtliche Bedeutung das Profil und der Score haben, hängt von den Folgen ab, die damit verbunden sind. Dabei ist danach zu unterscheiden, ob es eindeutige rechtliche Entscheidungen sind, die auf einem Score aufsetzen, ob es wirtschaftliche oder soziale Folgen sind, die durch das Handeln anderer entstehen, die den Score kennen, oder ob es automatisierte Entscheidungen sind, die die Folge herbeiführen. Die absehbaren Folgen beeinflussen auch die Zulässigkeit der Profil- und Score-Bildung (etwa bei der Bestimmung der schutzwürdigen Interessen). Rechtlich ergeben sich vor allem zwei Fragen, denen die folgenden Erwägungen nachgehen: Führt die quantifizierte Entscheidung zu einer unzulässigen Diskriminierung und darf eine automatisierte Entscheidung ergehen, die auf einem Profil oder einem Score beruht?

\subsection{Differenzierung und Diskriminierung}

Zahlen können sinnvolle Differenzierungen ausdrücken, sie können aber auch zu unerwünschten oder unzulässigen Diskriminierungen führen. Differenzierungen, die in bestimmten Zahlen ihren Ausdruck finden, können sozial oder individuell erwünscht sein. Um nicht jede Person zum Studium zuzulassen, ist es sozial erwünscht und rechtlich geregelt, dass für die Immatrikulation die Abiturnote berücksichtigt wird. Die Steuern, die jeder zu entrichten hat, hängen von der Steuerquote und dem Umsatz oder Einkommen ab. Konsumpunkte können bei der Bahn, der Airline oder Verkäufern zu Rabatten führen. Verkehrsverstöße können in der „Verkehrssünderdatei“ beim Kraftfahrt-Bundesamt in Flensburg zu Punkten führen, die am Ende über den Entzug der Fahrerlaubnis entscheiden. Individuell kann erwünscht sein, dass das Smart Car die Fahrerin erkennt und den Autositz so einstellt, dass alles passend ist. Im Smart Home soll beim Betreten die Musik laufen, die man gern hört, und die Temperatur so eingestellt sein, wie man sie gerne hätte. Selbst beim Datenschutz will eine betroffene Person, dass ein Programm, das sie im Datenschutz unterstützen soll, ihre Datenschutzpräferenzen kennt. Alle diese Anwendungen müssen auf Profile oder Scores zurückgreifen, um ihre sozial oder individuell gewünschten Funktionen zu erfüllen. Soweit der Wunsch oder die Notwendigkeit der Differenzierung besteht, ist die Datenverarbeitung gerechtfertigt und zulässig.

Es gibt aber auch Differenzierungen, die rechtlich verboten oder sozial unerwünscht sind. Nach Art. 3 Abs. 3 GG und Art. 21 GRCh sind für rechtlich relevante Entscheidungen Differenzierungen nach Geschlecht, Alter, Rasse, Behinderung, sexueller Orientierung, genetischen Merkmalen, ethnischer oder sozialer Herkunft, Sprache, Glauben, religiösen und politischen Anschauungen verboten. Sozial unerwünscht ist ene Differenzierung, wenn eine Orientierung 
am Durchschnitt statt an individuellen Merkmalen erfolgen soll. Soweit z.B. in Versicherungen das Solidaritätsprinzip gelten soll, sind bestimmte unterschiedliche Eigenschaften der Versicherten etwa für die Versicherungsprämie irrelevant.

Dies hat auch Auswirkungen auf die Verarbeitung personenbezogener Daten. Wenn bestimmte Merkmale keine Rolle spielen dürfen oder sollen, dürfen Daten über diese Eigenschaften auch nicht verarbeitet werden. Umgekehrt kann der Datenschutz auch die Differenzierungsmöglichkeiten einschränken. Soweit Datenschutzanforderungen wie Datenminimierung oder -löschung, Anonymisierung oder Pseudonymisierung umgesetzt werden, bestehen keine Daten über das individuelle Verhalten oder individuelle Merkmale. In diesen Fällen wird zum Schutz der informationellen Selbstbestimmung bewusst in Kauf genommen, dass Entscheidungen und Folgen nicht individualisiert erfolgen.

Wenn die Verarbeitung der Daten über unterschiedliche Eigenschaften von Menschen weder verboten noch unerwünscht noch unmöglich ist, stellt sich die Frage, ob eine Entscheidung legitimiert ist, die nach solchen Eigenschaften differenziert. Diese Frage betrifft die Anwendung des Gleichheitssatzes. Nach Art. 3 GG und Art. 20 GRCh sind ,,alle Personen ... vor dem Gesetz gleich“.

Diese Forderung kann aber nicht dazu führen, dass alle in allen Fragen gleich zu behandeln sind. Denn dies würde zu extremer Ungerechtigkeit führen. Aber auch die Umschreibung des Gleichheitssatzes, "Gleiches gleich und Ungleiches ungleich" zu behandeln, greift zu kurz. Denn alle Menschen haben gleiche (z.B. Menschwürde) und ungleiche Merkmale (z.B. eigene Geschichte). Letztlich kommt es auf das gewählte Unterscheidungsmerkmal an. Die entscheidende Frage ist also, ob es legitim ist, nach diesem Merkmal zu unterscheiden.

In der Beantwortung dieser Frage hat der Gesetzgeber einen weiten Entscheidungsspielraum. Insbesondere bei Massenerscheinungen (Steuer-, Sozial-, Verkehrsrecht) ist er befugt und bisweilen sogar verpflichtet, zu generalisieren, $\mathrm{zu}$ typisieren und $\mathrm{zu}$ pauschalieren. Auch darf er pauschaliert quantifizieren, ohne allein wegen damit verbundener Härten gegen den allgemeinen Gleichheitssatz zu verstoßen.

Für Unternehmen gelten die Grundrechte nicht unmittelbar. Vielmehr können sie sich selbst auf die Grundrechte der Handlungsfreiheit und der Berufsausübung sowie daraus abgeleitet auf die Vertragsfreiheit berufen. Aber auch sie müssen sich, je nach Umfang ihrer wirtschaftlichen und sozialen Macht, unterschiedlich stark an den Grundrechten orientieren. Ihre Vertragsfreiheit hat daher eine Grenze, wenn eine gewählte Unterscheidung sachfremd ist und willkürlich erscheint. Wann diese Grenze erreicht ist, bestimmt sich nach der Sozialadäquanz des Handelns oder nach den Kriterien eines fairen Verhaltens eines Kaufmanns. 
Bei lediglich verhaltensbezogenen Unterscheidungen hängen das Maß der Bindung an den Gleichheitssatz und die Intensität der Nachprüfung dieser Bindung davon ab, inwieweit die betroffene Person auf die Verwirklichung der Unterscheidungsmerkmale einwirken, durch entsprechendes Verhalten die nachteiligen Folgen also vermeiden kann. ${ }^{12}$ Ist ihr das ohne weiteres möglich, etwa durch ein bestimmtes rechtsgeschäftliches Verhalten, kommt eine Feststellung von Willkür nur in Betracht, wenn die Unterscheidung evident unsachlich ist. ${ }^{13}$ Das gilt auch für rein sachverhaltsbezogene Differenzierungen.

Das Diskriminierungsverbot kann verletzt sein, wenn etwa der Preis eines Produkts oder einer Dienstleistung von prognostizierten Eigenschaften einer Person abhängt - je nachdem, welche Merkmale der Person dieser Prognose zugrunde liegen. Willkürlich wäre es, wenn z.B. letztlich die ethnische oder religiöse Zugehörigkeit oder gesundheitliche Einschränkungen darüber entschieden, ob die Person von einer Dienstleistung oder einem Produkt ausgeschlossen, von ihr ein höherer Kaufpreis verlangt oder sie anderweitig schlechter gestellt wird (Roßnagel/Nebel 2015).

Die gleiche Frage nach einer legitimen oder illegitimen Unterscheidung ist zu stellen, wenn Scores genutzt werden, um automatisiert individuelle Preise zu entwickeln oder individuelle Vertragsbedingungen zu vereinbaren. Bei dynamischen Preisen dürfte es wohl weitgehend zulässig sein, diese zeitabhängig oder nachfrageabhängig zu gestalten. Werden sie individualisiert nach dem jeweiligen Score der Person gebildet, hängt die Frage einer zulässigen Ausübung der Vertragsfreiheit oder einer unzulässigen Willkür weitgehend davon ab, welche Merkmale dem Score zugrunde liegen. Jedenfalls aber fordert die notwendige Preistransparenz für Verbraucher nach § 5a UWG die Information darüber, dass es sich um individualisierte und dynamische Preise handelt.

\subsection{Automatisierte Entscheidungen}

Der Score kann die Grundlage einer automatisierten Entscheidung sein. Davor scheint Art. 22 Abs. 1 DSGVO die Verbraucherinnen zu bewahren. Nach dieser Vorschrift darf eine betroffene Person nicht einer ausschließlich auf einer automatisierten Verarbeitung beruhenden Entscheidung unterworfen werden, die ihr gegenüber rechtliche Wirkung entfaltet oder sie in ähnlicher Weise erheblich beeinträchtigt. Diese Regelung klingt im ersten Moment gut. Entscheidend ist jedoch weniger, was sie verbietet, sondern was sie gerade nicht verbietet. Sie hat - trotz der neuen erheblichen Herausforderungen durch Big Data, Künstliche Intelligenz, lernende Systeme und umfassendes Profiling - lediglich die

12 Ständige Rechtsprechung - s. z.B. BVerfG, Neue Juristische Wochenschrift 2014, 139.

13 BVerfG, Neue Zeitschrift für Arbeitsreche 1995,272. 
über 20 Jahre alte Regelung des Art. 15 Datenschutzrichtlinie nahezu wörtlich übernommen.

Die Regelung des Art. 22 Abs. 1 DSGVO unterscheidet zwischen der Datenverarbeitung und einer Entscheidung, die ,ausschließlich“ auf der automatisierten Verarbeitung beruht. Die der Entscheidung vorausgehende Datenverarbeitung wird von der Vorschrift nicht erfasst. Sie regelt nur den zweiten Schritt - die Entscheidung. Für die vorausgehende Datenverarbeitung gelten daher allein die allgemeinen risikoneutralen Erlaubnistatbestände des Art. 6 Abs. 1 und 4 DSGVO (s. Kap. 4.3). Diese gelten gleichermaßen für die Mitgliederliste eines kleinen Kegelklubs wie für das tiefgreifende Profiling der Nutzerpersönlichkeit durch globale Internetkonzerne (Roßnagel 2018). Die Vorschrift erfasst daher nicht die Steuerung von Geschäftsprozessen, den Einsatz von Legal Tech-Programmen oder die Erstellung von Persönlichkeitsprofilen und Scores. Vielmehr legitimiert sie durch den Zusatz ,-, einschließlich Profiling -“ diese Form der Verarbeitung personenbezogener Daten. Sie verbietet also nicht diese Persönlichkeitsanalysen, sondern akzeptiert sie für zulässige (nicht ausschließlich automatisierte) Entscheidungen.

Das Verbot des Art. 22 Abs. 1 DSGVO gilt außerdem nur für eine Entscheidung, die der betroffenen Person gegenüber „rechtliche Wirkung entfaltet oder sie in ähnlicher Weise erheblich beeinträchtigt". Erwägungsgrund 71 DSGVO nennt hierfür als Beispiele die ,automatische Ablehnung eines Online-Kreditantrags oder Online-Einstellungsverfahren ohne jegliches menschliche Eingreifen“. Entscheidungen mit rechtlicher oder ähnlich erheblicher Wirkung liegen dann vor, wenn sie Auswirkungen auf die Rechtsposition der betroffenen Person haben oder deren persönliche oder wirtschaftliche Entfaltung nachhaltig stören. Eine Anwendung des Verbots auf unerwünschte automatisierte Direktwerbung wird aus diesem Grund abgelehnt (Buchner 2018; Born 2015; Abel 2018). Das gleiche soll auch für die automatisierte Beschränkung von Zahlungsmöglichkeiten beim Online-Einkauf, für die Ablehnung eines Vertragsabschlusses oder die Verweigerung bestimmter Vertragskonditionen gelten (Schulz 2018). Ob dies auch für verhaltensbedingte Werbung oder individuelle Preisgestaltung gilt, hängt von dem vagen Begriff der ,in ähnlicher Weise erheblichen Beeinträchtigung" ab.

Das Verbot gilt auch dann nicht, wenn das Profil oder der Score die Entscheidungsvorlage für eine menschliche Entscheidung ist - selbst wenn der Mensch mangels eigener Kenntnis oder Zeit nicht in der Lage ist, die automatisiert vorgeschlagene Entscheidung zu hinterfragen, und in allen Fällen wie maschinell vorgeschlagen entscheidet.

Weiterhin greift das Verbot nicht, soweit ein Verantwortlicher, etwa ein Anbieter im E-Commerce, automatisierte Entscheidungen anderer zur Voraussetzung für den Abschluss oder die Erfüllung eines Vertrags zwischen der betrof- 
fenen Person und ihm macht. Erklärt etwa eine Bank die Feststellung ausreichender Bonität oder Liquidität der betroffenen Person durch eine Auskunftei zur Voraussetzung für einen Kreditvertrag, ist die automatisierte Festlegung eines Kredit-Scores nach Art. 22 Abs. 2 lit. a DSGVO zulässig.

Schließlich kommt Art. 22 DSGVO nach seinem Abs. 2 lit. b nicht zur Anwendung, wenn die Mitgliedstaaten anderes geregelt haben. Eine solche Regelung hat die Bundesrepublik Deutschland als Ergebnis erfolgreicher Lobbyarbeit in $\S 37$ BDSG zugunsten von Versicherungen getroffen.

Insgesamt ist daher festzustellen, dass Art. 22 DSGVO nur ein Teilproblem und dieses unzureichend erfasst. Den Herausforderungen durch Profiling und Scoring in einer Welt des Big Data, des Ubiquitous Computing und der Künstlichen Intelligenz wird diese Vorschrift nicht gerecht.

In der Praxis wichtiger als Art. 22 DSGVO ist für zwei bestimmte Verwendungsweisen von Profilen $§ 31$ BDSG. Die Vorschrift definiert Scoring als Verwendung eines Wahrscheinlichkeitswerts über ein bestimmtes zukünftiges Verhalten einer natürlichen Person zum Zweck der Entscheidung über die Begründung, Durchführung oder Beendigung eines Vertragsverhältnisses mit dieser Person. Nach Abs. 1 dieser Vorschrift ist Scoring nur zulässig, wenn die Vorschriften des Datenschutzrechts eingehalten wurden und die zur Berechnung des Wahrscheinlichkeitswerts genutzten Daten unter Zugrundelegung eines wissenschaftlich anerkannten mathematisch-statistischen Verfahrens nachweisbar für die Berechnung der Wahrscheinlichkeit des bestimmten Verhaltens erheblich sind. Die Vorschrift legt außerdem fest, dass für das Scoring nicht ausschließlich Anschriftendaten genutzt werden dürfen. Sollen diese neben anderen Daten genutzt werden, muss die betroffene Person vor Berechnung des Wahrscheinlichkeitswerts über die vorgesehene Nutzung dieser Daten in dokumentierter Weise unterrichtet worden sein.

$\S 31$ Abs. 2 BDSG enthält Regelungen für die Verwendung von Scores durch Auskunfteien für die Vorhersage der Zahlungsfähig- und Zahlungswilligkeit einer natürlichen Person. Die Berechnung der Wahrscheinlichkeitswerte darf nur bestimmte Informationen über Forderungen einbeziehen wie z.B. gerichtlich festgestellte oder von der Schuldnerin ausdrücklich anerkannte Forderungen.

\section{Quantifizierte Verhaltenssteuerung}

Das Wissen über Menschen, das aus Profiling stammt und durch Scoring leicht verfügbar wird, kann dazu genutzt werden, das Verhalten von Individuen oder Kollektiven zu beeinflussen oder gar zu steuern. Dies kann zu Beeinträchtigun- 
gen der Selbstbestimmung und Selbstentfaltung führen, wird aber vom Recht kaum erfasst.

\subsection{Microtargeting und Nudging}

Das Wissen aus Profiling und Scoring kann genutzt werden, um Menschen gezielt $\mathrm{zu}$ beeinflussen. Individuelle Profile ermöglichen, einzelne Personen in Form eines Microtargeting direkt, gezielt und automatisiert anzusprechen. Durch eine entsprechende Wahl der Informationen kann ein mehr oder weniger starker Druck zu bestimmten Verhaltensweisen erzeugt werden. Diese Informationen können Verhaltensweisen als Voraussetzung für die der betroffenen Person wichtige soziale Anerkennung oder als Grund für befürchtete soziale Ausgrenzung erscheinen lassen. Umgekehrt können auch an spezifische Präferenzen ansetzende ,unwiderstehliche“ Angebote beinahe zwanghaft wirken, da ihnen die betroffene Person kaum ausweichen kann.

Diese Formen des individuellen Nudging können auch für kommerzielle Anwendungen, insbesondere im Bereich der Werbung, genutzt werden. Erkennt ein Smartphone z.B., dass sein Nutzer sich gerade in einer euphorischen Stimmung befindet, bietet dieses Wissen die Möglichkeit, ihm passgenau in diesem Moment Werbung und eine Bestellmöglichkeit für etwas einzublenden, von dem man vermutet, dass der Nutzer es mögen könnte. Profiling und Scoring kann also helfen zu erkennen, wann bei einem bestimmten potentiellen Kunden (oder Wähler) besonders wenige hemmende Faktoren für eine erwünschte Willensentscheidung vorliegen und wie sich solche Faktoren bei diesem Individuum umgehen lassen (Roßnagel/Geminn/Jandt/Richter 2016; Hoffmann-Riem 2017).

Im Ergebnis können geeignete Informationsimpulse aus der Ferne und automatisiert direkt und im vielversprechendsten Augenblick in den Prozess der individuellen Willensbildung eingreifen. Dies beeinträchtigt die in Art. 2 Abs. 1 GG verbürgte Willens- und Handlungsfreiheit.

\subsection{Normativität der Normalität}

Aber selbst dann, wenn die Daten anonym verarbeitet werden, also kein individueller Score vorliegt, ist eine ungezielte Beeinflussung des Verhaltens möglich und sogar wahrscheinlich. Allein statistische Daten haben verhaltensbeeinflussende Wirkungen. Sie beschreiben das normale Verhalten. Diese Normalität hat normative Wirkung. Wenn bekannt wird, dass die überwiegende Mehrheit sich in einer bestimmten Weise verhält und dieses „Normalverhalten“ bestimmten Entscheidungen zugrunde gelegt wird, werden sich die meisten nach dieser erwarteten Normalität verhalten, um die gewünschten Wirkungen zu erzielen. 
Statistische Muster wirken dadurch wie soziale Regeln. Auch in diesem Fall bemerken die wenigsten Menschen, dass sie durch die Art der Informationen, die ihnen präsentiert werden, fremdbestimmt werden.

Durch die Mustererkennung, wie sie z.B. Big Data-Analysen ermöglichen, wirken Muster sozialen Verhaltens normbildend. Die Muster korrelieren Verhaltensmerkmale und beschreiben „normales“ und „abweichendes“ Verhalten. Wenn an diese Muster positive und negative Entscheidungen anknüpfen, werden sich die Menschen diesen Mustern anpassen, um in den Genuss der positiven Wirkungen zu gelangen und negative zu vermeiden. Verhaltensmuster können durch diese Normbildung indirekt, aber wirkungsvoll die Wahrnehmung von Grundrechten beeinflussen. Die anonymen Muster wirken so genauso negativ auf die Persönlichkeitsentfaltung des Einzelnen und die freie Kommunikation und Willensbildung in der Gesellschaft insgesamt ein, wie dies das Bundesverfassungsgericht bereits im Volkszählungsurteil als Auswirkungen personenbezogener Überwachung festgestellt hat. ${ }^{14}$

Die informationelle Selbstbestimmung kann keinen Schutz gegen die normierende Wirkung von statistischen Verhaltensmustern bieten, soweit hierfür keine personenbezogenen Daten verwendet werden. Dennoch können sie die Grundrechtsausübung und das demokratische Engagement gefährden (Weichert 2013; Roßnagel 2013). Durch das Einordnen des Verhaltens in statistische Handlungsmuster als konform oder nicht konform und durch das so indirekt erzwungene Anpassungsverhalten werden die Entscheidungs- und die Verhaltensfreiheit faktisch eingeschränkt, was das Recht auf informationelle Selbstbestimmung gerade vermeiden soll. Solche statistischen Muster verstärken die Normativität der Normalität und reduzieren „Soziodiversität“. Diese ist aber eine Voraussetzung für Innovationen und Demokratie (Roßnagel/Nebel 2015).

\subsection{Gefangen in der Statistik}

Doch auch wer sich gegen diese Quantifizierung stemmt und sich selbstbestimmt für einen eigenen Lebensstil entscheidet, wird zum Opfer des Prinzips der anonymen Vergemeinschaftung. Jeder wird über die statistisch erfassten Merkmale einer für ihn anonymen Gemeinschaft (Hubig 2008) zugeordnet und nach deren statistischem Muster behandelt. Werden Infrastrukturen, Waren und Dienstleistungen an den statistischen Mustern orientiert, sind ihre Angebote den Merkmalen der Zielgruppe angepasst. Selbstbestimmte Entscheidungen werden dadurch sowohl unmöglich als auch irrelevant. 
Keiner kann sich der Statistik verschließen: Auch wer sich weigert, seine Daten für statistische Zwecke herzugeben, unterliegt dennoch der auch ohne ihn entstehenden Statistik. Diese fordert nicht die Teilnahme aller, sie funktioniert auch, wenn nur repräsentativ viele mitwirken. Die Verweigerung einzelner nutzt nichts. Die Statistik gilt auch für die Verweigerer. Selbstbestimmung ist in diesem Fall zwecklos. Auch wer keine Daten zur Verfügung gestellt hat, wird etwa hinsichtlich seiner Kreditwürdigkeit nach dem Durchschnitt der Gruppe bemessen, auf die die meisten seiner Merkmale (z.B. Alter, Geschlecht, Familienstand, Beruf, Wohnort) passen (Roßnagel 2016).

\section{Rechtspolitische Schlussfolgerungen}

$\mathrm{Zu}$ allen vier Untersuchungsthemen sind Ergebnisse festzuhalten und rechtspolitische Schlussfolgerungen zu ziehen:

Die Menschenwürde fordert Schutz vor einer Verwandlung des Menschen zum reinen Informationsobjekt, dessen Leben Informationslieferant für die unbegrenzte Verhaltenssteuerung ist. Die zunehmende Transparenz des Menschen gegenüber den großen Datenverarbeitern und die steigende Intransparenz der Datenverarbeitung verstärken die soziale Machtasymmetrie. Solche Informationsmachtbildung gefährdet die Demokratie. Schutz der Menschwürde und Schutz der Demokratie fordern klare - demokratisch legitimierte - Grenzen für die Verarbeitung personenbezogener Daten.

Die Quantifizierung der Persönlichkeit durch Profiling und Scoring gefährdet Selbstbestimmung und Selbstentfaltung. Unabhängig davon, ob auf deren Grundlage automatisch oder durch eine Person entschieden wird, sind spezifische Regeln für legitime Zwecke, Umfang, Verwendung und Qualität von Profilen und Scores erforderlich.

Profiling, Scoring und statistische Musterbildung verstärken und erzeugen neue soziale Ungleichheiten. Die Frage, welche Differenzierungen erwünscht sind und welche Diskriminierung unterbleiben soll, dürfen weder die Technik (selbstlernende Systeme) noch private Verantwortliche entscheiden. Über diese gesellschaftsrelevante Frage müssen legitimierte soziale Instanzen entscheiden. Sie müssen festlegen, wo das Verursacherprinzip mit der Folge der Individualisierung oder wo das Solidaritätsprinzip mit der Folge des Verzichts auf personenbezogene Daten gelten soll.

Profiling, Scoring und statistische Muster gefährden die Entscheidungsfreiheit der Individuen und die Willensbildung in einer demokratischen und freien Gesellschaft. Um sie zu schützen, sind Regelungen notwendig, die unerwünschtes gezieltes Nudging und indirekte Verhaltenssteuerung durch statistische Methoden kontrollieren und verhindern. 
Für eine freie, demokratische Gesellschaft, die auf die selbstbestimmte und ungezwungene Mitwirkung ihrer Bürger angewiesen ist, ist eine Einschränkung der Grundrechte durch die Quantifizierung der Persönlichkeit nicht akzeptabel. Es ist daher Teil der staatlichen Schutzpflicht, die ungehinderte Ausübung der Grundrechte zu gewährleisten.

\section{Literatur}

Abel, R. B. (2018). Automatisierte Entscheidungen im Einzelfall gem. Art. 22 DS-GVO. Anwendungsbereich und Grenzen im nicht-öffentlichen Bereich, Zeitschrift für Datenschutz, 304 308.

Ammicht Quinn, R. u.a. (2018): Tracking - Beschreibung und Bewertung neuer Methoden, White Paper des Forums Privatheit und selbstbestimmtes Leben in der digitalen Welt, Karlsruhe.

Born, T. (2015). Bonitätsprüfungen im Online-Handel. Scorewert-basierte automatisierte Entscheidung über das Angebot von Zahlungsmöglichkeiten, Zeitschrift für Datenschutz 66-70.

Brink, S./Hertfelder, S. (2019). Einwilligung und Vertragsdatenverarbeitung, in: Roßnagel, A./ Hornung, G. (Hrsg.), Grundrechtsschutz im Smart Car, Berlin/Wiesbaden, Springer Verlag, $75-86$.

Buchner, B. (2018). Kommentierung des Art. 22 DSGVO, in: Kühling, J./Buchner, B. (Hrsg.), Datenschutz-Grundverordnung mit Bundesdatenschutzgesetz, 2. Aufl. München: Beck Verlag.

Di Fabio, U. (2001). Kommentierung des Art. 2 GG, in: Maunz, T./Düring, G. (Hrsg.), GG-Kommentar, 39. EL, München: Beck Verlag.

Dzida, B./Groh, N. (2018). Algorithmen im Bewerbungsverfahren, Neue Juristische Wochenschrift, 1917-1922.

Herdegen, M. (2018). Kommentierung des Art. 1 GG, in: Maunz, T./Düring, G. (Hrsg.), GG-Kommentar, 83. EL, München: Beck Verlag.

Hoffmann-Riem, W. (2017). Verhaltensteuerung durch Algorithmen - Eine Herausforderung für das Recht, Archiv des öffentlichen Rechts, 142. Band, 1-42.

Hornung, G./Engemann, C. (2016). Der digitale Bürger und seine Identität, Baden-Baden: Nomos Verlag.

Hubig, C. (2008). Der technisch aufgerüstete Mensch - Auswirkungen auf unser Menschenbild, in: Roßnagel, A./Sommerlatte, T./Winand, U. (Hrsg.), Digitale Visionen - Zur Gestaltung allgegenwärtiger Informationstechnologien, Berlin: Springer Verlag 2008, 165-177.

Jarass, H. D. (2018). Kommentierung des Art. 1 GG, in: Jarass, H. D./Pieroth, B. (Hrsg.), GGKommentar, 15. Aufl. München: Beck Verlag.

Jarass, H. D. (2016). Kommentierung des Art. 1 GRCh, in: Jarass, H. D. (Hrsg.), GRCh-Kommentar, 3. Aufl. München: Beck Verlag.

Kunig, P. (2012). Kommentierung des Art. 1 GG, in: von Münch, I./Kunig, P (Hrsg.), GG-Kommentar, 6. Aufl. München: Beck Verlag.

Moos, F./Rothkegel, T. (2016). Nutzung von Scoring-Diensten im Online-Versandhandel. ScoringVerfahren im Spannungsfeld von BDSG, AGG und DS-GVO, Zeitschrift für Datenschutz, $561-565$. 
Roßnagel, A. (2013). Big Data - Small Privacy? Konzeptionelle Herausforderungen für das Datenschutzrecht, Zeitschrift für Datenschutz, 566-570.

Roßnagel, A. (2016). Datenschutz: Eine Zukunft ohne Selbstbestimmung? Spektrum der Wissenschaft kompakt online vom 4.10.2016, 41-49.

Roßnagel, A. (2018). Das künftige Datenschutzrecht in Europa, in: ders. (Hrsg.), Das neue Datenschutzrecht, Baden-Baden: Nomos Verlag.

Roßnagel, A. (2019). Kommentierung von Art. 5 DSGVO, in: Simitis, S./Hornung, G./Spiecker, I. (Hrsg.), Datenschutzrecht - DSGVO - BDSG, Baden-Baden: Nomos Verlag.

Roßnagel, A./Geminn, C. L./Jandt, S./Richter, P. (2016). Datenschutzrecht 2016 - „Smart“ genug für die Zukunft? Ubiquitous Computing und Big Data als Herausforderungen des Datenschutzrechts, Kassel: kassel university press.

Roßnagel, A./Nebel, M. (2015). (Verlorene) Selbstbestimmung im Datenmeer - Privatheit im Zeitalter von Big Data, Datenschutz und Datensicherheit, 455-460.

Sachverständigenrat für Verbraucherfragen (SVRV) (2018): Verbrauchergerechtes Scoring, Berlin: Sachverständigenrat für Verbraucherfragen.

Schulz, S. (2018). Kommentierung des Art. 22 DSGVO, in: Gola, P. (Hrsg.), DSGVO, 2. Aufl. München: Beck Verlag.

Taeger, J. (2016). Scoring in Deutschland nach der EU-Datenschutz-Grundverordnung, Zeitschrift für Rechtspolitik, 72-76.

Weichert, T. (2013). Big Data und Datenschutz. Chancen und Risiken einer neuen Form der Datenanalyse, Zeitschrift für Datenschutz, 258-263. 
